\title{
Comparison of the performance
} of the CareStart Malaria Pf/Pan Combo test and field microscopy in the diagnosis of Plasmodium vivax malaria in North Sumatera, Indonesia

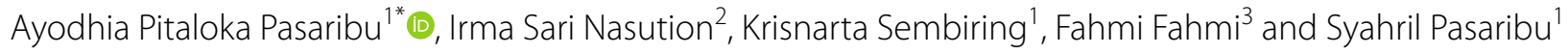

\begin{abstract}
Background: In areas where malaria is endemic and where trained microscopists are not available, rapid diagnostic tests (RDTs) are needed not only to allow prompt treatment without delay but also to prevent overdiagnosis and overtreatment based on clinical judgements that may lead to drug resistance. This study aimed to compare the performances of the CareStart Pf/Pan Combo test to field microscopy, which is considered to be the gold standard for malaria diagnosis.
\end{abstract}

Methods: Any person with a fever or a history of fever within $48 \mathrm{~h}$ who came to the health centre was recruited for the study and tested both by the CareStart Pf/Pan test and by field microscopy. Sensitivity, specificity, positive predictive value, negative predictive value, and accuracy were analysed with both methods.

Results: Two-hundred study participants were enrolled: 96 (48\%) were found to be positive through microscopy, while 100 (50\%) participants were found to be positive through RDT. The RDT produced four false-positive results. High sensitivity and specificity were observed for the CareStart Pf/Pan test (100 and 96.15\%, respectively). The CareStart Pf/Pan test also showed excellent agreement with the field microscopy results.

Conclusion: The Carestart Pf/Pan could be used as an alternative diagnostic test in malaria-endemic areas where facility for performing microscopy is not available.

Keywords: CareStart, Field microscopy, Malaria, Rapid diagnostic test, Indonesia

\section{Background}

Malaria remains a leading cause of morbidity and mortality worldwide, with an estimated 229 million new cases and 409,000 deaths annually [1]. As in other countries in Southeast Asia, Indonesia aims to achieve

*Correspondence: ayodhiapp@usu.ac.id

${ }^{1}$ Department of Child Health, Medical Faculty, Universitas Sumatera Utara, Medan 20155, Indonesia

Full list of author information is available at the end of the article malaria elimination by 2030 , and scaling up diagnostics and treatment interventions is currently essential [2]. In areas where malaria transmission has declined, accurate diagnosis is very important, not only for case management but also for disease surveillance $[3,4]$. Clinical signs and symptoms of malaria are non-specific, which may result in overdiagnosis and inappropriate treatment using anti-malarial drugs that can lead to the selection of drug-resistant variants $[4,5]$. The main strategy for malaria control of the World Health Organization 
(WHO) is to improve diagnosis by detecting parasites by either microscopy or by a rapid diagnostic test (RDT) [6]. Microscopy remains the gold standard for malaria diagnosis because it is inexpensive and can provide information on the specific parasite species and parasite density [7].

However, microscopy is time consuming and requires trained personnel, careful preparations and a constant supply of power for operating equipment, which is limited in most areas where malaria is endemic [8]. RDTs could be used as an alternative method for the diagnosis of malaria in areas lacking microscopes or trained technicians and allows for laypersons to conduct the test after limited training [9]. Over 60 different malaria RDTs have geographical areas $[10,11]$. Factors such as ambient conditions, variability of the detected antigen and different parasite densities may influence the performance of the tests [10]. Several previous studies found that the CareStart Pf/Pan combo test has a higher sensitivity for Plasmodium falciparum than for Plasmodium vivax compared to microscopy $[12,13]$.

For $P$. vivax, pan-lactate dehydrogenase ( $\mathrm{pLDH})$ tests showed sensitivities ranging from 62.0 to $95.0 \%$ [12-14]. Polymerase chain reaction (PCR) allows low densities of malaria parasites to be detected in blood samples. It is important for epidemiological and clinical research especially for sub-microscopic recurrences, however, it should not be used in diagnosis and management as it is too sensitive [15].

This study was conducted to evaluate the performance of the CareStart RDT Pf/Pan combo test for the detection of $P$. vivax compared to field microscopy, which is the gold standard, in the Tanjung Leidong subdistrict, North Sumatra, Indonesia. This strategy is in line with the recent Malaria National Strategic Plan for 2020-2024 for Indonesia that recommends regular evaluations with RDTs within the country [16]. The hypothesis was that the CareStart Pf/Pan combo test has a comparable performance to field microscopy for the diagnosis of $P$. vivax.

\section{Methods}

\section{Study area and design}

The cross-sectional study comparing RDT to field microscopy was conducted at Tanjung Leidong Health Centre in Kualuh Leidong District, North Labuhan Batu Regency, from July to December 2018, which was a peak transmission season for malaria in the area. The district covers an area of $394 \mathrm{sq} \mathrm{km}$ with a population of more than 29,552, the majority of which depends on fishing (Fig. 1) [17]. Tanjung Leidong Health Centre offers malaria diagnosis and treatment for free. Plasmodium vivax is the predominant vector species in this location.

\section{Sample size}

To detect $P$. vivax with a sensitivity of $80 \%$ (alpha error 0.1 , precision 5\%), 83 blood smear-positive and 83 blood smear-negative samples were required. The detection of malaria-carrying species was limited to $P$. vivax.

\section{Study procedure}

In inclusion criteria, any person with a fever or a history of fever within $48 \mathrm{~h}$ who visited the health centre was selected as a study participant. As for exclusion criteria, individuals who took anti-malarial drugs within the four weeks prior to the study or refused to participate were excluded. Both CareStart Pf/Pan combo tests and thick and thin blood smear microscopic examinations were simultaneously performed on samples obtained from each patient with acute febrile illness.

The diagnostic performance of the CareStart Pf/Pan combo test targeting the pLDH antigen was evaluated against the performance of thick blood-smear microscopy. Microscopy is still the gold standard for diagnosing malaria, thus under field condition it may miss some symptomatic cases, however it is still suitable for clinical diagnosis [15]. The health centre staff members were trained on selecting acute febrile cases and collecting samples for laboratory diagnosis. Explanations regarding the study were given to the participants before the samples were collected. Thick and thin blood smears were prepared on the same slide; the thin blood smears were fixed with methanol, and the thick smears were unfixed. Each slide was then stained with a 3\% Giemsa solution for $45 \mathrm{~min}$. All blood smears were examined microscopically under an oil immersion lens at $1000 \times$ magnification. For positive smears, the numbers of parasites were counted against 200 white blood cells (WBCs) in thick smears or $500 \mathrm{WBCs}$ for low-density infections. Parasite density was calculated assuming $8000 \mathrm{WBCs}$ per $\mu \mathrm{l}$ (18) [18]. The thin smears for the positive samples were examined to confirm the species identification. All sample slides included in this study were examined by a single field microscopist who was blinded to the RDT results. All positive slides were sent to Eijkman Institute, Jakarta, for quality control. The RDT kit used was the CareStart Malaria HRP-2/pLDH (Pf/Pan) combo test (Access Bio, Belgium). This kit is a three-band RDT targeting HRP-2 and pan-pLDH.

The CareStart tests used in this study were donated by the Ministry of Health. Test kits were stored at room temperature and the expiration date was checked before use. The tests were performed according to the directions of the manufacturer. The CareStart tests were labelled with a patient identification number and date, and the results were recorded after $20 \mathrm{~min}$. The presence 


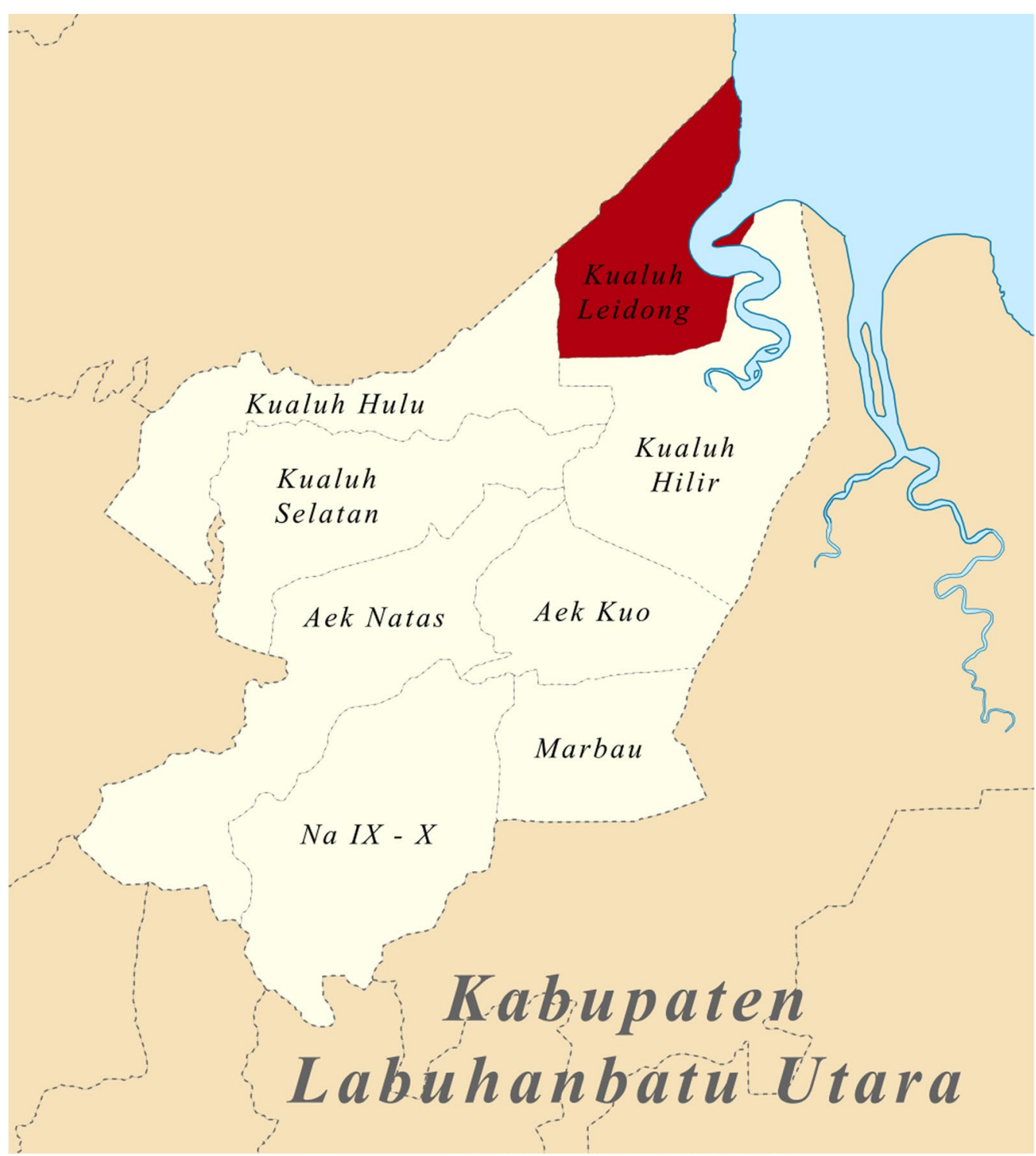

Fig. 1 Map of the study site. https://commons.wikimedia.org/wiki/Category:Kualuh_Leidong,_Labuhanbatu_Utara

of a unique pan-pLDH line is found when an individual is infected with one or more non-falciparum species. A test result without a control line was considered invalid, and the sample was retested. All the data were doubleentered by study staff to maintain accuracy.

\section{Data analysis}

Data were entered and analysed using STATA version 15.1 (Stata Corporation, College Station, TX, USA) after checking for completeness. Data were analysed for descriptive statistics, while results of both methods were compared using Cohen's kappa co-efficient. The sensitivity, specificity, positive predictive value (PPV), and negative predictive value (NPV) were calculated. All statistical analyses were done at 95\% level of significance.

\section{Results}

During the study period, 207 patients with fever were screened and tested for malaria with the CareStart Pf/ Pan test and field microscopy, among which 200 patients agreed to participate. The other seven patients refused to participate. More than one-half of the study participants were male (55.5\%). By field microscopy, 96 (48\%) study participants were found to be positive for $P$. vivax infection, while $100(50 \%)$ tested positive with the CareStart $\mathrm{Pf} / \mathrm{Pan}$ test. The only species detected in the positive 
blood smears and RDTs was $P$. vivax. The baseline characteristics are shown in Table 1.

Using microscopy as the gold standard test for detecting malaria by $P$. vivax infection, the sensitivity and specificity of the CareStart Pf/Pan combo test were 100\% (95\% CI 96.23-100) and 96.15\% (95\% CI 90.18-98.43), respectively. There were four (2\%) discordant results obtained between field microscopy and the CareStart Pf/Pan test. All the discordant results were negative in the field microscopy test and positive in the CareStart Pf/Pan test. Unfortunately, the discordant samples were not tested to PCR as a confirmatory test that would help improve the reliability of the test.

The PPV and the NPV of the CareStart Pf/Pan test were determined to be $98 \%$ (95\% CI 94.96-99.45) and $100 \%$, respectively. The agreement of the results between the CareStart Pf/Pan tests and the field microscopy tests were excellent, with a kappa value of 0.96 (Table 2). The results of the quality control tests performed at the Eijkman Institute showed that 4 of the 96 field microscopypositive samples were negative. The sensitivity of field microscopy compared to the Eijkman Institute readings was 97.87\% (95\% CI 92.52-99.74) and the PPV was 95.83\% (95\% CI 89.79-98.37). The readings at the Eijkman Institute differed from the field microscopy because of the level of expertise; the Eijkman Institute is a reference laboratory with expert readers, while in the field there was a basic reader.

The CareStart Pf/Pan test revealed an increasing number of positive results with increasing levels of
Table 2 Performance of the CareStart Pf/Pan test compared to the gold standard, field microscopy

\begin{tabular}{|c|c|c|}
\hline Field microscopy & CareStart Pf/Pan & \\
\hline & Positive & Negative \\
\hline Positive & 96 & 0 \\
\hline Negative & 4 & 100 \\
\hline Sensitivity, \% (95\% Cl) & $100(96.23-100)$ & \\
\hline Specificity, \% (95\% Cl) & 96.15 (90.44-98.99) & \\
\hline PPV, \% (95\% Cl) & $96(90.18-98.40)$ & \\
\hline NPV, \% (95\% Cl) & $100(N / A)$ & \\
\hline Accuracy, \% (95\% Cl) & $98(94.96-99.45)$ & \\
\hline Cohen's kappa (95\% Cl) & $0.96(0.92-0.99)$ & \\
\hline
\end{tabular}

parasitaemia, with parasitaemia $>1000 / \mu$, accounting for $91.67 \%$ of all positive RDT results (Fig. 2).

\section{Discussion}

RDTs for malaria diagnosis are vital to make prompt treatment decisions, especially at healthcare facilities that do not have trained microscopists $[18,19]$. Even though a detailed assessment of the performance had been undertaken by $\mathrm{WHO} /$ Foundation for innovative diagnostic (FIND), it is important to evaluate the tests in different endemic area settings and as performed by health workers involved in the daily healthcare routine [20,21]. In this study, the only malaria-causing parasite observed was $P$. vivax. This finding was similar to the study performed at the same site in 2013 [22] but differed from

Table 1 Baseline characteristics

\begin{tabular}{|c|c|}
\hline \multicolumn{2}{|l|}{ Study participant characteristics ( $\mathrm{n}=200$ ) } \\
\hline \multicolumn{2}{|l|}{ Sex, n (\%) } \\
\hline Female & $89(44.5)$ \\
\hline Male & $111(55.5)$ \\
\hline \multicolumn{2}{|l|}{ Age classification, n (\%) } \\
\hline$<18$ years & $81(40.5)$ \\
\hline$>18$ years & $119(59.5)$ \\
\hline Mean hemoglobin level, g/dL (SD) & $12.0(1.83)$ \\
\hline Median temperature, ${ }^{\circ} \mathrm{C}$ (range) & $38.0(36.4-40.2)$ \\
\hline \multicolumn{2}{|l|}{ RDT result, $\mathrm{n}(\%)$} \\
\hline Positive & $100(50.0)$ \\
\hline Negative & $100(50.0)$ \\
\hline \multicolumn{2}{|l|}{ Microscopic examination result, n (\%) } \\
\hline Positive & $96(48.0)$ \\
\hline Negative & $104(52.0)$ \\
\hline \multicolumn{2}{|l|}{ Parasitological characteristics $(n=96)$} \\
\hline Geometric mean asexual density parasite/ $\mu \mathrm{L}(95 \% \mathrm{Cl})$ & $2717.47(2143.39-3445.32)$ \\
\hline 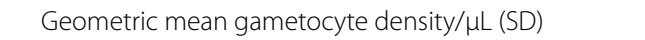 & $2128(1195.71)$ \\
\hline
\end{tabular}




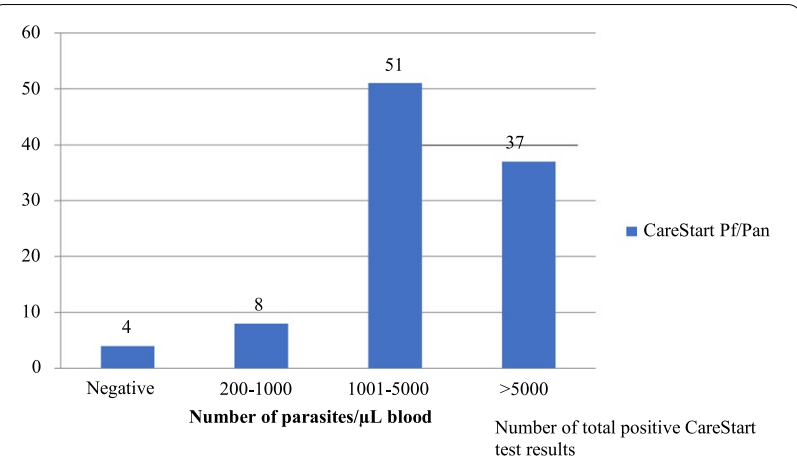

Fig. 2 Number of positive CareStart Pf/Pan test results, categorized into different $P$. vivax densities

findings obtained in Nigeria, where Plasmodium falciparum was the only observed parasite [21]. The majority of the study participants in the present study were male and older than 18 years, which was similar to the cohort in previous studies conducted in Ethiopia and at the ChinaMyanmar border [23, 24].

A study performed in Korea found that one patient tested negative by RDT (SD BIOLINE), while the microscopy result was positive, which was a result similar to that of a study performed in Ethiopia with the CareStart combo test $[25,26]$. These findings were different from those of the present study, where four study participants tested positive by the CareStart Pf/Pan test but negative by field microscopy. Several reasons for this result might be due to other infectious diseases or the presence of rheumatoid factors that were not assessed in this study [27]. Another reason could be attributable to PLDH production by plasmodial gametocytes, which may result in false-positive RDT results [28]. The overall diagnostic performance of the CareStart Pf/Pan test when compared to that of field microscopy was very good in the present study. The sensitivity and specificity of the CareStart Pf/ Pan test in this study were 100 and $96.15 \%$, respectively. These results were similar to several studies that were conducted in different areas $[18,19]$ but higher than reported studies conducted in Ethiopia [25].

Interestingly, the high sensitivity of the CareStart Pf/ Pan test in this study was different to that in a study from Sumba, Indonesia, where the sensitivity of the test was only $13.6 \%$ [29]. The higher sensitivity might have been the result of the high number of parasites found in the current study's samples ( $>200$ parasites $/ \mu \mathrm{L}$ ). The high positive and negative predictive values in this study were similar to the findings of Feleke and Moges obtained in northwestern Ethiopia [25, 30]. The sensitivity could not be categorized based on different parasite densities because the overall sensitivity was $100 \%$; however, an increasing number of positive RDT results was found with increasing parasitaemia. Several studies using the CareStart RDT reported higher sensitivity with higher parasitaemia $[23,24]$ but another study performed in South Korea presented different results [31]. This study also observed excellent agreement between the CareStart Pf/Pan test results and the field microscopy results, which was similar to other studies [24].

Limitations of this study included the cross-sectional design that limited the detection of sub-microscopic parasites that may have been found in a follow-up test. In the present study, only the CareStart Pf/Pan test results were compared with field microscopy results; however, the true burden of sub-microscopic infection would have been better determined by polymerase chain reaction (PCR) and new diagnostic tests should have the ability to detect sub-microscopic infections to achieve malaria elimination [32].

Nevertheless, the comparison of RDT and microscopy results for malaria diagnosis is seldom conducted in Indonesia, and the study results are important because they are in line with the current Malaria National Strategic Plan for 2020-2024.

\section{Conclusion}

The CareStart Pf/Pan test showed very good sensitivity and specificity with excellent agreement with the results obtained with use of the gold standard, field microscopy. This result provides important information to the Ministry of Health.

\section{Abbreviations}

HRP: Histidine rich protein; pLDH: Pan-lactate dehydrogenase; PCR: Polymerase chain reaction; RDT: Rapid diagnostic test; WBC: White blood cell; WHO: World Health Organization.

\section{Acknowledgements}

The authors are grateful to all the patients who participated in this study. The authors thank the staff of the Tanjung Leidong Health Centre and the Head of North Labuhan Batu for their contributions. Special thanks are due to Mr Dian Syahputra, who helped with the microscopic examinations and Ms Farah Coutrier from the Eijkman Institute for performing quality control evaluations of the microscopy readings.

\section{Authors' contributions}

Conceived the study: APP, SP. Designed the experiments: APP, FF. Performed the experiments: APP, ISN. Analysed the data: APP, KS. Critically reviewed and contributed to writing: APP, ISN, KS, FF, SP. All authors read and approved the final manuscript.

\section{Funding}

This project was supported by the Directorate General of Research and Development Strengthening, the Ministry of Research, the Technology and Higher Education of The Republic of Indonesia (No:135/UN5.2.3.1/PPM/ KP-DRPM/2018). The content of this study is solely the responsibility of the 
authors and does not necessarily represent the official views of the funding agencies.

\section{Availability of data and materials}

The data used to support the findings of this study are available from the corresponding author upon request.

\section{Declarations}

\section{Ethics approval and consent to participate}

The study was approved by the Ethical Committee of the Medical Faculty Universitas Sumatera Utara/Adam Malik General Hospital No. 588/TGL/KEPK FK USU-RSUP HAM/2018. Written informed consent was obtained from each of the study participants, including from the parents and guardians of the children who were included.

\section{Consent for publication}

Not applicable.

\section{Competing interests}

The authors declare that there are no competing interests regarding the publication of this paper.

\section{Author details}

'Department of Child Health, Medical Faculty, Universitas Sumatera Utara, Medan 20155, Indonesia. ${ }^{2}$ Medical Faculty, Universitas Sumatera Utara, Medan 20155, Indonesia. ${ }^{3}$ Department of Electrical Engineering, Universitas Sumatera Utara, Medan 20155, Indonesia.

Received: 22 May 2021 Accepted: 19 January 2022

Published online: 29 January 2022

\section{References}

1. WHO. World malaria report 2015. Geneva: World Health Organization; 2020.

2. Sitohang V, Sariwati E, Fajariyani SB, Hwang D, Kurnia B, Hapsari RK, et al. Malaria elimination in Indonesia: halfway there. Lancet Glob Health. 2018:6:e604-6.

3. malERA Consultative Group of Diagnoses and Diagnostics. A research agenda for malaria eradication: diagnoses and diagnostics. PLoS Med. 2011;8:e1000396

4. McMorrow ML, Aidoo M, Kachur SP. Malaria rapid diagnostic tests in elimination settings-can they find the last parasite? Clin Microbiol Infect. 2011:17:1624-31.

5. Leslie T, Mikhail A, Mayan I, Anwar M, Bakhtash S, Nader M, et al. Overdiagnosis and mistreatment of malaria among febrile patients at primary healthcare level in Afghanistan: observational study. BMJ. 2012;345:e4389.

6. World Global Malaria Programme. World malaria report 2010. Geneva: World Health Organization; 2011.

7. WHO. Malaria microscopy quality assurance manual, version II. Geneva: World Health Organization; 2016.

8. Moyeh MN, Ali IM, Njimoh DL, Nji AM, Netongo PM, Evehe MS, et al. Comparison of the accuracy of four malaria diagnostic methods in a high transmission setting in coastal Cameroon. J Parasitol Res. 2019:2019:1417967.

9. Gerstl S, Dunkley S, Mukhtar A, De Smet M, Baker S, Maikere J. Assessment of two malaria diagnostic tests in children under five years of age, with follow-up of false-positive pLDH test results, in a hyperendemic falciparum malaria area, Sierra Leone. Malar J. 2010;9:28.

10. Murray CK, Bennett JW. Rapid diagnosis of malaria. Interdiscip Perspect Infect Dis. 2009;2009:415953.

11. WHO. Malaria rapid diagnostic tests: what is RDT? Accuracy of actions. Geneva: World Health Organization; 2005.

12. Maltha J, Gillet P, Bottieau E, Cnops L, van Esbroeck M, Jacobs J. Evaluation of a rapid diagnostic test (CareStart Malaria HRP-2/pLDH (Pf/pan) Combo Test) for the diagnosis of malaria in a reference setting. Malar J. 2010;9:171.
13. Mekonnen Z, Ali S, Belay G, Suleman S, Chatterjee S. Evaluation of the performance of CareStart Malaria Pf/Pv Combo rapid diagnostic test for the diagnosis of malaria in Jimma, southwestern Ethiopia. Acta Trop. 2010;113:285-8.

14. Fogg C, Twesigye R, Batwala V, Piola P, Nabasumba C, Kiguli J, et al. Assessment of three new parasite lactate dehydrogenase (pan-pLDH) tests for diagnosis of uncomplicated malaria. Trans R Soc Trop Med Hyg. 2008;102:25-31.

15. Chu CS, White NJ. The prevention and treatment of Plasmodium vivax malaria. PLoS Med. 2021;18:e1003561.

16. WHO. National malaria programme review Republic of Indonesia. Jakarta: Kementerian Kesehatan REpublik Indonesia; 2021. p. 39-44.

17. Kualuh Leidong, Labuhanbatu Utara. https://id.wikipedia.org/wiki/ Kualuh_Leidong,_Labuhanbatu_Utara. Accessed 08 Jan 2022.

18. WHO. Basic malaria microscopy-part I: learner's guide. 2 nd ed. Geneva: World Health Organization; 2010.

19. Singh N, Shukla MM, Shukla MK, Mehra RK, Sharma S, Bharti PK, et al. Field and laboratory comparative evaluation of rapid malaria diagnostic tests versus traditional and molecular techniques in India. Malar J. 2010;9:191.

20. Hailu T, Kebede T. Assessing the performance of CareStart Malaria Pf/ Pv Combo Test against thick blood film in the diagnosis of malaria in northwest Ethiopia. Am J Trop Med Hyg. 2014;90:1109-12.

21. Wogu MN, Nduka FO. Evaluating malaria prevalence using clinical diagnosis compared with microscopy and rapid diagnostic tests in a tertiary healthcare facility in Rivers State. Nigeria J Trop Med. 2018;2018:3954717.

22. Pasaribu AP, Chokejindachai W, Sirivichayakul C, Tanomsing N, Chavez I, Tjitra E, et al. A randomized comparison of dihydroartemisinin-piperaquine and artesunate-amodiaquine combined with primaquine for radical treatment of vivax malaria in Sumatera, Indonesia. J Infect Dis. 2013;208:1906-13.

23. Ashton RA, Kefyalew T, Tesfaye G, Counihan H, Yadeta D, Cundill B, et al. Performance of three multi-species rapid diagnostic tests for diagnosis of Plasmodium falciparum and Plasmodium vivax malaria in Oromia Regional State, Ethiopia. Malar J. 2010;9:297

24. Xiaodong S, Tambo E, Chun W, Zhibin C, Yan D, Jian W, et al. Diagnostic performance of CareStart ${ }^{\mathrm{TM}}$ malaria HRP2/pLDH (Pf/pan) combo test versus standard microscopy on falciparum and vivax malaria between China-Myanmar endemic borders. Malar J. 2013;12:6.

25. Feleke DG, Tarko S, Hadush H. Performance comparison of CareStart ${ }^{\mathrm{TM}}$ HRP2/pLDH combo rapid malaria test with light microscopy in north-western Tigray, Ethiopia: a cross-sectional study. BMC Infect Dis. 2017:17:399.

26. Kim KH, Jang JW, Woo MK, Oh JS, Han ET, Lee WJ, et al. Evaluation of four rapid diagnostic tests for the diagnosis of Plasmodium vivax in Korea. Trop Med Int Health. 2011;16:1427-31.

27. Watson OJ, Sumner KM, Janko M, Goel V, Winskill P, Slater HC, et al. False-negative malaria rapid diagnostic test results and their impact on community-based malaria surveys in sub-Saharan Africa. BMJ Glob Health. 2019:4:e001582.

28. Wongsrichanalai C, Barcus MJ, Muth S, Sutamihardja A, Wernsdorfer WH. A review of malaria diagnostic tools: microscopy and rapid diagnostic test (RDT). Am J Trop Med Hyg. 2007;77(6 Suppl):119-27.

29. Ahmed R, Levy El, Maratina SS, de Jong JJ, Asih PB, Rozi IE, et al. Performance of four HRP-2/pLDH combination rapid diagnostic tests and field microscopy as screening tests for malaria in pregnancy in Indonesia: a cross-sectional study. Malar J. 2015;14:420.

30. Moges B, Amare B, Belyhun Y, Tekeste Z, Gizachew M, Workineh M, et al. Comparison of CareStart ${ }^{\mathrm{TM}} \mathrm{HRP} 2 / \mathrm{pLDH}$ COMBO rapid malaria test with light microscopy in north-west Ethiopia. Malar J. 2012;11:234

31. Kim JY, Ji SY, Goo YK, Na BK, Pyo HJ, Lee HN, et al. Comparison of rapid diagnostic tests for the detection of Plasmodium vivax malaria in South Korea. PLOS ONE. 2013;8:e64353.

32. Nguyen TN, von Seidlein L, Nguyen TV, Truong PN, Hung SD, Pham HT, et al. The persistence and oscillations of submicroscopic Plasmodium falciparum and Plasmodium vivax infections over time in Vietnam: an open cohort study. Lancet Infect Dis. 2018;18:565-72.

\section{Publisher's Note}

Springer Nature remains neutral with regard to jurisdictional claims in published maps and institutional affiliations. 